\title{
CONTROL OF SHELL SHAPE IN LYMNAEA STAGNALIS
}

\author{
WALLACE ARTHUR \\ Department of Biology, Sunderland Polytechnic, Sunderland SR1 3SD, U.K.
}

Received 28.iv. 82

\section{SUMMARY}

\begin{abstract}
A component of shell shape was measured in two populations of the freshwater gastropod Lymnaea stagnalis, and a highly significant difference noted. Samples of juveniles were then taken from both natural populations, and cultured in the laboratory in tanks containing water from the pond inhabited by one of the natural populations; that is, one of the laboratory populations can be regarded as a transplant, the other as a control. These two cultures were maintained until substantial growth of the juvenile snails had occurred. The shell shapes of the snails were then measured and the two populations found to be almost identical. The difference between this comparison and the one involving the two natural populations was caused entirely by a marked shift in the shell shape distribution of the transplanted population. Thus the large difference in shape observed between the populations in the wild was completely due to direct environmental effects on the phenotype, or any genetic component of the variation was so small as to be undetectable by the method employed. It is noted that this result cautions against acceptance of the evolutionary inferences that are often drawn from studies of phenotypic variation in shell shape where these are unaccompanied by demonstration of an inherited component of the variation.
\end{abstract}

\section{INTRODUCTION}

STUDIES of phenotypic variation of gastropod shells are widespread and evolutionary inferences are often drawn from them. The pulmonates, both freshwater and terrestrial, have been particularly often studied and the characters examined include both those with discrete variants and those whose variation is usually continuous (see review by Clarke et al., 1978). While the genetic basis of the former type of variation is often well understood (for example, pigmentation in Cepaea: Murray, 1975), the genetic control of continuous variation in shell characters, and indeed whether such variation is inherited at all, usually remains obscure. As long as this is the case, evolutionary inferences drawn from studies based on continuously-variable shell characters are suspect and alternative nongenetic interpretations of patterns of temporal or spatial variation remain possible. This is problematical, especially since studies based on continuously-variable morphological characters of gastropod shells have been used to support a wide range of evolutionary theories including punctuated equilibrium (Williamson, 1981; several genera of gastropods and bivalves), character displacement (Fenchel, 1975; Hydrobia) and genetically differentiated geographical races (Mozley 1935, 1939; Lymnaea).

Some of these studies were based on shell size while others concerned shell shape. There have been several investigations of the heritability of shell size (Cook, 1965, 1967-Arianta and Cepaea; Murray and Clarke, 1968-Partula) but the heritability of shape has received relatively little 
recent attention, with the exception of Newkirk and Doyle's (1975) study of three measures of shell shape in Littorina. There is thus a need for further work on the degree of genetic and environmental control of shell shape variation within and between populations of gastropod species. Here I report a study of the control of variation in shape between populations of the freshwater pulmonate Lymnaea stagnalis which is a particularly variable species.

\section{Methods}

\section{(i) Sampling sites}

The natural habitats sampled were: Site 1 -a stagnant pond separated from the southern shore of Lough Neagh, Northern Ireland, by a strip of land about $5 \mathrm{~m}$ wide; Site 2-an inlet to the north-western corner of Lough Neagh close to the New University of Ulster's field station at Traad Point. Grid references are J0264 and H9689 respectively; the two sites are approximately $30 \mathrm{~km}$ apart. Maximum diameters of the sites were $10 \mathrm{~m}$ (site 1) and $50 \mathrm{~m}$ (site 2). Since the sample from site 2 consisted of two sub-samples from slightly separated sub-areas within the site, the data on these sub-samples $(H / A$ values: see below) were compared, revealing a negligible and non-significant difference, before being pooled.

\section{(ii) Measurement of shell shape}

Two measurements that can be made on the shell of $L$. stagnalis or, for that matter, on the shell of most gastropods, are: $H$, the height of the shell from the lower rim of the lip to the apex; and $A$, the aperture height, measured at its maximum span (and so not necessarily exactly parallel to the measurement of $H$ ). These characters were both measured, to the nearest $0.1 \mathrm{~mm}$, using vernier calipers. The simplest description of shape that can be employed is a ratio involving two distinct measurements, and in the present study the ratio $H / A$ was used. This aspect of shape has also been examined in some early studies of variation in shell shape in Lymnaea: Piaget (1928, 1929), Mozley (1935).

\section{(iii) Experimental design}

Samples of $L$. stagnalis were taken from the two sampling sites and their shell-shape distributions analysed. The difference in shape between the two samples was highly significant (see Results section). Juvenile snails (shell height approximately $5 \mathrm{~mm}$ ) were then taken from both sites and grown for a two-month period in identical tanks in the laboratory; the tanks both contained water taken from site 1 . At the end of this period, $H / A$ distributions were analysed. One potential problem with this design should be noted-it may underestimate the environmental component of the variation due to the founders of the laboratory populations being juveniles rather than eggs. As will be seen, the results of the experiment were such that this problem can be eliminated. 

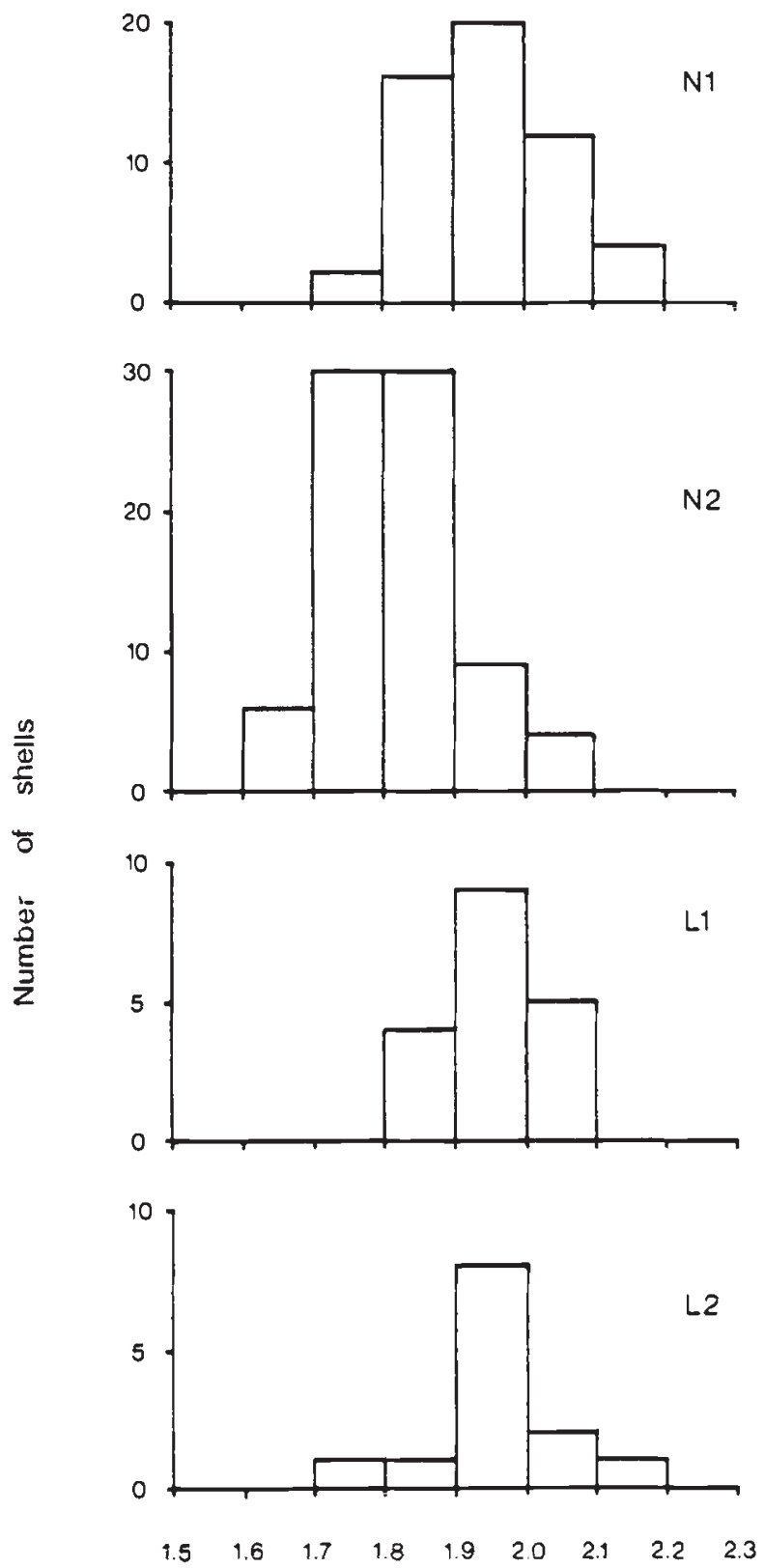

$H / A$

FIG. 1. $-H / A$ distributions for samples from natural populations $(N 1, N 2)$ and for laboratory populations $(L 1, L 2)$. 


\section{Results}

The $H / A$ distributions for all four populations are shown in fig. 1 ; the means, standard deviations and confidence limits for these distributions are given in table 1. Differences in mean $H / A$ value were tested for significance using $t$-tests and the results of this analysis are shown in table 2 . The large and very highly significant difference between the samples from the two natural populations is clearly removed by laboratory culturing since populations $L 1$ and $L 2$ are almost identical in mean $H / A$ value. The difference between the $N 1 / N 2$ comparison and the $L 1 / L 2$ comparison is caused entirely by a shift in the $H / A$ values of the transplanted $L 2$ population which is significantly different from its donor (N2) population but not detectably different from either of the populations $(N 1, L 1)$ with which it shared a common water source.

TABLE 1

Descriptive statistics of $H / A$ distributions

\begin{tabular}{|c|c|c|c|c|}
\hline Population & $n$ & Mean & $\begin{array}{c}H / A \\
\text { Standard deviation }\end{array}$ & $95 \%$ Confidence limits \\
\hline$N 1$ & 54 & 1.949 & $0 \cdot 090$ & $1.925-1.973$ \\
\hline$N 2$ & 79 & $1 \cdot 816$ & 0.092 & $1.795-1.837$ \\
\hline$L 1$ & 18 & 1.954 & 0.070 & $1.919-1.989$ \\
\hline$L 2$ & 13 & 1.946 & $0 \cdot 100$ & $1 \cdot 886-2.006$ \\
\hline
\end{tabular}

$N 1, N 2$ Natural populations of sites 1 and 2 respectively.

$L 1, L 2$ Laboratory-reared populations founded by juveniles taken from sites 1 and 2 respectively.

TABLE 2

Pairwise comparisons of mean $H / A$ values

$\begin{array}{cccc}\text { Comparison between } & t & \text { df } & P \\ N 1 \text { and } N 2 & 8.365 & 131 & <0.001 \\ L 1 \text { and } L 2 & 0.261 & 29 & \text { ns } \\ L 1 \text { and } N 1 & 0.216 & 70 & \text { ns } \\ L 1 \text { and } N 2 & 6.012 & 95 & <0.001 \\ L 2 \text { and } N 1 & 0.106 & 65 & \text { ns } \\ L 2 \text { and } N 2 & 4.676 & 90 & <0.001\end{array}$

The most obvious interpretation of these results is that the large interpopulation difference in shape observed in nature was due entirely to direct environmental effects on the phenotype. However, two potential complicating factors need to be considered before this conclusion can be confirmed. The first of these is the possibility that $H / A$ co-varies with $H$, which is important in the present context since the shells in $L 1$ and $L 2$ were considerably smaller than those in $N 1$ and $N 2$. Since gastropod shells of ten approximate to an equiangular spiral (Thompson, 1942) there should be no problem in that the increase of $H$ and $A$ with growth should be isometric rather than allometric. However, it has been pointed out (e.g., by Gould, 1971) that the shells of some species are not a good approximation to the equiangular spiral, and so it is necessary to check the validity of comparing $H / A$ values for shells of different sizes. This has been done for populations $N 1, N 2, L 1$ and $L 2$ separately and for the data on $L 1$ and $N 1$ combined. 
TABLE 3

Results of tests for correlation between $\mathrm{H} / \mathrm{A}$ and $\mathrm{H}$

$\begin{array}{cccc}\text { Population } & r & \mathrm{df} & P \\ N 1 & +0 \cdot 085 & 52 & \mathrm{~ns} \\ N 2 & +0 \cdot 361 & 77 & \simeq 0 \cdot 001 \\ L 1 & -0 \cdot 174 & 16 & \text { ns } \\ L 2 & -0 \cdot 445 & 11 & \text { ns } \\ N 1+L 1 & -0 \cdot 005 & 70 & \text { ns }\end{array}$

(The data on $L 2$ and $N 2$ cannot be combined as the populations experienced different environments). The results of analyses of correlation between $H / A$ and $H$ are summarized in table 3 , and the data for $N 1 / L 1$ and for $N 2$ are illustrated in figs 2 and 3 respectively. It can be seen that only in $N 2$ is there a significant association between the two variables.

This positive correlation of $H$ and $H / A$ in population $N 2$ does not render the results of the $t$-test analysis ambiguous because it is in the wrong direction to provide an alternative explanation for the shift in $H / A$ values of population $L 2$, as compared with $N 2$. In fact, a Model II regression of the $N 2$ data yields an $H / A$ prediction of 1.705 for an $H$-value of $12.06 \mathrm{~mm}$ (the mean shell height for population $L 2$ ). Thus the fact that $L 2$ shows an increased mean $H / A$ value (1.946) that is almost identical to those of $N 1$ and $L 1$ is even more remarkable than if $H / A$ and $H$ were uncorrelated.

The second potential complicating factor is that selection may act in experiments of the kind reported here and its effects may be mistaken for direct environmental effects. In this context it should be noted that the small numbers in populations $L 1$ and $L 2$ were due to few juveniles being available in the natural populations at the time the laboratory cultures

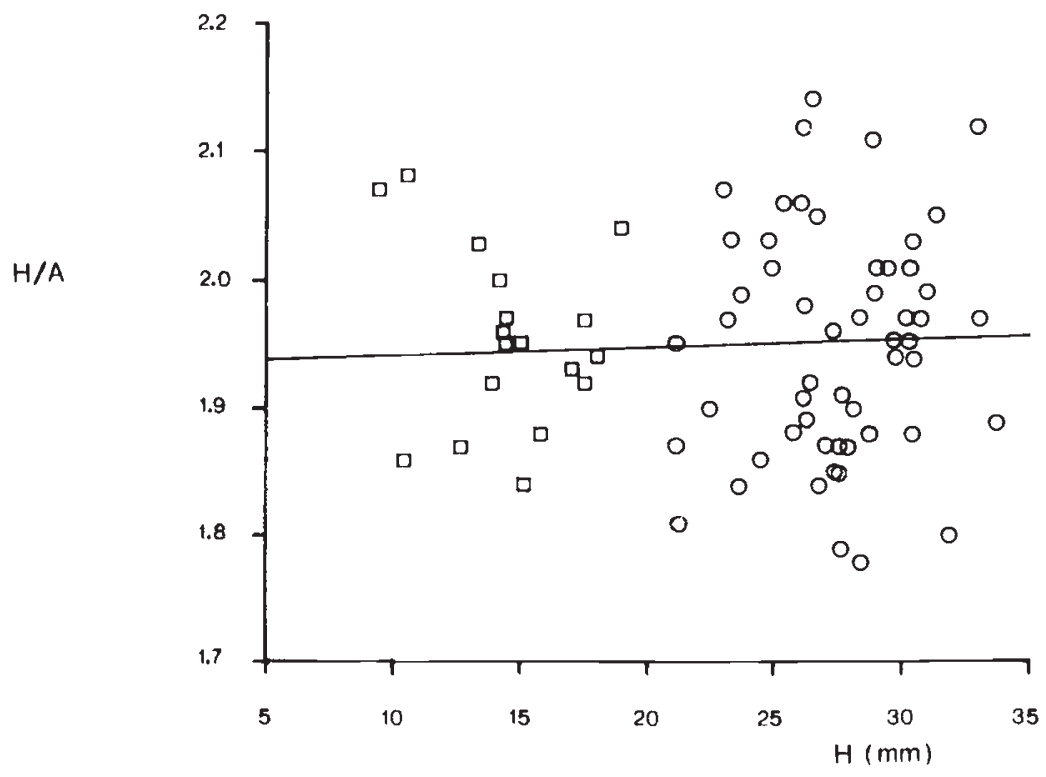

Fig. 2.-Scattergram of $H / A$ against $H$ for combined data of $N 1(O)$ and $L 1$ (口). Model II regression line is described by the equation $y=0.0007 x+1.934$. 


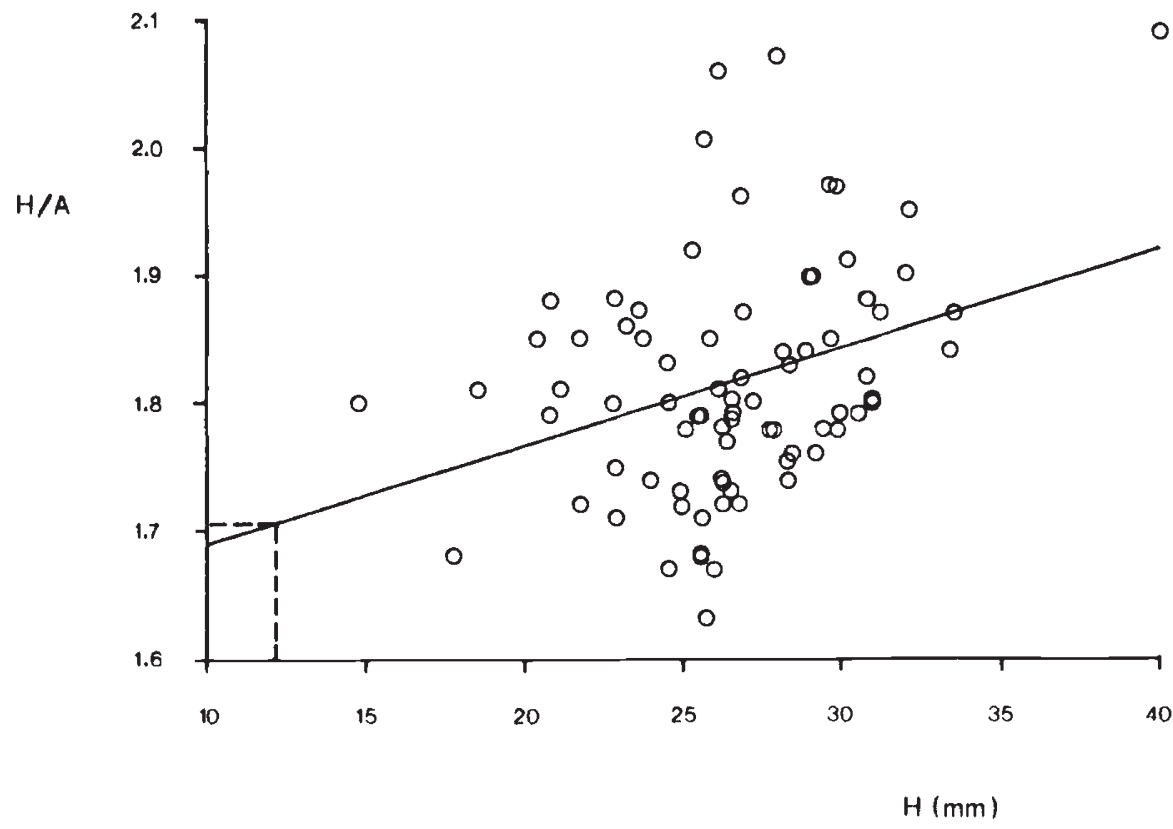

Fig. 3.-Scattergram of $H / A$ against $H$ for $N 2$ data. Model II regression line is described by the equation $y=0.0077 x+1.611$. The dashed lines show the predicted $H / A$ value $(1.705)$ corresponding to the mean shell height $(12.06 \mathrm{~mm})$ for population $L 2$.

were set up; and not to heavy mortality in these cultures (only one snail died in each). This fact is important since it excludes the possibility of selection operating in the $L 2$ laboratory cultures and causing the shift in $\boldsymbol{H} / \boldsymbol{A}$ values observed in that population.

\section{Discussion}

The data presented in the previous section show a particularly clear case of environmental control of shell shape variation between two populations. However, the question arises as to how typical this conclusion is of variation in shape between other populations of $L$. stagnalis and, for that matter, between populations of other pulmonate species. Work on the pulmonates in general has been discussed by Clarke et al. (1978) and the discussion below will be largely restricted to studies of species of Lymnaea.

The first study of the control of variation in shell shape in Lymnaea appears to have been that of Roszkowski (1914) who worked on the "species" known as $L$. profunda and $L$. abyssicola found at considerable depth in Lac Léman, Switzerland. Roszkowski regarded these as variant forms of $L$. ovata (now $L$. peregra) and $L$. palustris respectively, and indeed he found, on laboratory culturing of both forms, reversion to the "typical" $L$. peregra and $L$. palustris shell shape in the first generation. Thus the forms that had earlier been named as profunda and abyssicola, because of their aberrant shell shapes, differed from their respective species norms due merely to a direct effect of the deep-lake environment on individual development. 
Piaget (1928, 1929), working with L. stagnalis, obtained a completely different result. It appears (Piaget, 1929) that variant forms of $L$. stagnalis taken from different localities were genetically divergent in that differences in shell shape persisted, despite identical laboratory conditions, for five generations. This conclusion is not entirely certain, partly due to an odd form of data presentation (frequency curves for $H / A$ where all laboratory generations for a particular "race" were pooled), and partly since, as Clarke et al. (1978) point out, such experiments do not exclude the action of cytoplasmically transmitted agents. Nevertheless, the difference between Piaget's results and those of Roszkowski is striking. One possible explanation of this difference (Waddington, 1975) is that in some populations a squat shell is an environmentally-induced feature while in others it has become genetically assimilated.

Boycott (1938) bred various forms of $L$. peregra in laboratory culture for several generations and found that some forms altered to "typical" $L$. peregra in a single generation (i.e., similar to Roszkowski's results) while others remained constant for several generations, as in Piaget's experiments. Moreover, these differing outcomes were in one case obtained in the same variant form ("involuta") from different localities.

The early work on the control of shell shape in Lymnaea suffers from two main problems. One is the frequent lack of quantitative measurements and consequent lack of statistical analysis; much of the work was based on descriptions and photographs of the shells involved. Also, the water used in the laboratory cultures was usually not taken from one of the natural habitats but from some convenient source. Thus, for example, Boycott (1938) used hard water in his laboratory cultures whereas the natural populations sampled inhabited mountain lakes with soft water. Boycott's expectation of a particular shell shape in his laboratory cultures was based on the assumption that calcium concentration was important in the determination of shape. However, while this may well be true, various other environmental agents as disparate as parasitic mites (Oldham, 1931) and wave action (Newkirk and Doyle, 1975) can affect shell shape in gastropods. Thus it is possible to set up a laboratory environment which unwittingly mimics some important aspect of the natural environment from which the cultured snails came. Constancy of shell shape over several generations in such cultures need not indicate a genetic contribution to the variation. Thus it is useful to transfer snails from one locality to a laboratory culture consisting of water from another population's locality-i.e., "transplant" rather than "implant" experiments-in order to obtain a clearer prediction of the form of shape alteration to be expected if direct environmental effects occur. Also, since some of the factors capable of modifying shell shape are not chemical agents in the water, it is desirable, in laboratory transplant experiments, to simulate physical and biotic conditions also, as far as possible. Hence the present experiment was conducted in the appropriate "direction" so that water from the stagnant pond (Site 1) was used in the laboratory cultures rather than water from Site 2. The latter site was much more subject to wave motion, which would be difficult to reproduce in the laboratory.

Despite the enormous variation in shell shape that is now known in various species of Lymnaea (see Hubendick, 1951) the present study appears to be the first investigation of the control of this variation using a 
quantitative estimate of shape (together with appropriate analyses) and involving a transplant rather than an implant experiment. The results clearly indicate a large direct environmental influence on shell shape. However, it is desirable that further studies be conducted to see whether reports such as Piaget's (1929) of populations differing genetically in shell shape can be confirmed. Other work that needs to be done includes estimation of heritability of shape within populations, estimation of the degree of self-and cross-fertilization and the effect of geographic variation in this aspect of reproductive biology on patterns of genetic and non-genetic variation in shape, and comparison of results for different estimates of shape and for different species. Studies of these kinds are now being planned.

While statements on the generality of any mode of causation of shell shape variation await the results of these additional studies, the existence of any cases, such as the one described here, where large differences in shell shape between populations turn out to be entirely, or almost entirely, environmental in origin, is problematical from an evolutionary viewpoint. It means that, unless accompanied by a demonstration of at least some genetic component, studies of phenotypic variation in shell shape cannot be used as clear evidence of evolutionary patterns. (Note that it is information on the inheritance of differences between populations that is needed to clarify whether a pattern of interpopulation variation represents the product of some regime of selection, whereas it is the heritability of variation within populations that relates to their future evolutionary potential.) There is no reason why information on the control of shell shape cannot be provided in the case of most microevolutionary studies since usually a single generation is all that is required. Certainly in the absence of such information, statements (Mozley, 1935; Lymnaea) that variation in $H / A$ values are under genetic control cannot be accepted. In macroevolutionary studies of variation in shell morphology such as that of Williamson (1981; see also Boucot, 1982) information on the control of shell shape of course cannot be obtained for the populations involved. However, information on the control of shell morphology in the nearest living relatives of the populations whose evolution is studied would be a considerable advance on no information at all.

Acknowledgements. - I would like to thank Professor A. Macfadyen and Mr T. Andrew for their very helpful suggestions and discussion during the course of the work, and Dr J. S. Jones for comments and criticism of the manuscript.

\section{REFERENCES}

BOUCOT, A. J. 1982. Ecophenotypic or genotypic? Nature, 296, 609-610.

BOYCOTT, A. E. 1938. Experiments on the artificial breeding of Limnaea involuta, Limnaea burnetti and other forms of Limnaea peregra. Proc. malac. Soc. Lond., 23, 101-108.

CLARKE, B., ARTHUR, W., HORSLEY, D. T., AND PARKIN, D. T. 1978. Genetic variation and natural selection in pulmonate molluscs. In Fretter, V. and Peake, J. (eds.) Pulmonates, vol. 2A, Academic Press, London.

COOK, L. M. 1965. Inheritance of shell size in the snail Arianta arbustorum. Evolution, 19, 86-94.

COOK, L. M. 1967. The genetics of Cepaea nemoralis. Heredity, 22, 397-410.

FENCHEL, T. 1975. Character displacement and coexistence in mud snails (Hydrobiidae). Oecologia, 20, 19-32. 
GOULD, S. J. 1971. Environmental control of form in land snails. A case of unusual precision. Nautilus, 84, 86-93.

HUBENDICK, B. 1951. Recent Lymnaeidae. Their variation, morphology, taxonomy, nomenclature and distribution. Kungl. Svenska Vetenskaps Akad. Handl., (4) 3, 1-223.

MOZLEY, A. 1935. The variation of two species of Lymnaea. Genetics, 20, 452-465.

MOZLEY, A. 1939. The variation of Lymnaea stagnalis (Linné). Proc. malac. Soc. Lond., 23, 267-269.

MURRAY, J. 1975. The genetics of the Mollusca. In King, R. C. (ed.) Handbook of Genetics, vol. 3, Plenum, New York.

MURRAY, J., AND ClARKE, B. 1968. Inheritance of shell size in Partula. Heredity, 23, 189-198.

NEWKIRK, G. F., AND DOYLE, R. W. 1975. Genetic analysis of shell-shape variation in Littorina saxatilis on an environmental cline. Marine Biology, 30, 227-237.

OLDHAM, C. 1931. Some scalariform examples of Arianta arbustorum infested by parasitic mites. Proc. malac. Soc. Lond., 19, 240-242.

PIAGET, J. 1928. Un problème d'hérédité chez la limnée des étangs. Appel aux malacologistes et aux amateurs en conchyliologie. Bull. Soc. Zool. Fr., 53, 13-18.

PIAGET, J. 1929. Les races lacustres de la Lymnaea stagnalis L. Bull. Biol. France Belg., 63, 424-455.

ROSZKOWSKI, w. 1914. Contribution a l'étude des limnées du Lac Léman. Rev. Suisse Zool., 22, 457-539.

THOMPSON, D'A. W. 1942. On Growth and Form, second edition. Cambridge University Press, Cambridge.

WADDINGTON, C. H. 1975. The Evolution of an Evolutionist. Edinburgh University Press, Edinburgh.

WILliAMSON, P. 1981. Palaeontological documentation of speciation in Cenozoic molluscs from Turkana Basin. Nature, 293, 437-443. 